\title{
Revisiting the hydration structure of aqueous $\mathrm{Na}^{+}$
}

\author{
M. Galib, ${ }^{1}$ M. D. Baer, ${ }^{1}$ L. B. Skinner, ${ }^{2}$ C. J. Mundy, ${ }^{1}$ T. Huthwelker ${ }^{3}$ G. K. Schenter,${ }^{1}$ \\ C. J. Benmore ${ }^{2}$ N. Govind ${ }^{4}$ and J. L. Fulton $\left.{ }^{1, a}\right)$ \\ ${ }^{1}$ Physical Sciences Division, Pacific Northwest National Laboratory, Richland, Washington 99354, USA \\ ${ }^{2} X$-ray Science Division, Advanced Photon Source, Argonne National Laboratory, Argonne, Illinois 60439, USA \\ ${ }^{3}$ Swiss Light Source, Paul Scherrer Institute (PSI), 5232, Villigen, Switzerland \\ ${ }^{4}$ Environmental Molecular Sciences Laboratory, Pacific Northwest National Laboratory, Richland, Washington \\ 99354, USA
}

(Received 28 November 2016; accepted 23 January 2017; published online 27 February 2017)

A combination of theory, X-ray diffraction (XRD) and extended x-ray absorption fine structure (EXAFS) are used to probe the hydration structure of aqueous $\mathrm{Na}^{+}$. The high spatial resolution of the XRD measurements corresponds to $\mathrm{Q}_{\max }=24 \AA^{-1}$ while the first-reported Na K-edge EXAFS measurements have a spatial resolution corresponding to $2 \mathrm{k}=\mathrm{Q}_{\max }=16 \AA^{-1}$. Both provide an accurate measure of the shape and position of the first peak in the $\mathrm{Na}-\mathrm{O}$ pair distribution function, $\mathrm{g}_{\mathrm{NaO}}(\mathrm{r})$. The measured $\mathrm{Na}-\mathrm{O}$ distances of $2.384 \pm 0.003 \AA$ (XRD) and $2.37 \pm 0.024 \AA$ (EXAFS) are in excellent agreement. These measurements show a much shorter $\mathrm{Na}-\mathrm{O}$ distance than generally reported in the experimental literature $\left(\mathrm{Na}-\mathrm{O}_{\text {avg }} \sim 2.44 \AA\right.$ ) although the current measurements are in agreement with recent neutron diffraction measurements. The measured $\mathrm{Na}-\mathrm{O}$ coordination number from XRD is $5.5 \pm 0.3$. The measured structure is compared with both classical and first-principles density functional theory (DFT) simulations. Both of the DFT-based methods, revPBE and BLYP, predict a $\mathrm{Na}-\mathrm{O}$ distance that is too long by about $0.05 \AA$ with respect to the experimental data (EXAFS and XRD). The inclusion of dispersion interactions (-D3 and $-\mathrm{D} 2)$ significantly worsens the agreement with experiment by further increasing the $\mathrm{Na}-\mathrm{O}$ distance by $0.07 \AA$. In contrast, the use of a classical Na-O Lennard-Jones potential with SPC/E water accurately predicts the $\mathrm{Na}-\mathrm{O}$ distance as $2.39 \AA$ although the $\mathrm{Na}-\mathrm{O}$ peak is over-structured with respect to experiment. Published by AIP Publishing. [http://dx.doi.org/10.1063/1.4975608]

\section{INTRODUCTION}

Sodium is the sixth most abundant element on earth. The low ionization enthalpy and relatively low polarizability of sodium make the cation, $\mathrm{Na}^{+}$, the most common ionic species in aqueous solution. $\mathrm{Na}^{+}$plays a role in a broad spectrum of biochemical, geochemical, and industrial chemistry. It is for these reasons that $\mathrm{Na}^{+}$has been the primary subject of hundreds of research articles over the last decades. The deceptively simple electronic configuration of sodium belies the complexity of its solvation in water that is, to a large extent, controlled by the nuanced properties of the water-water interactions. It is essential to fully understand the delicate balance of forces that define the $\mathrm{Na}^{+}$hydration structure and the shortand long-range structure of this cation with various anionic species.

A literature survey of the theoretical ${ }^{1-4}$ and experimen$\mathrm{tal}^{5-9}$ results shows a large uncertainty of $2.44 \pm 0.1 \AA$ for the position of the first peak in the $\mathrm{Na}-\mathrm{O}$ pair distribution function. This is significant because the magnitude of this error leads to (i) large uncertainties in the hydration enthalpies and (ii) to uncertainties in the size of ion-water frameworks that are important in ion transport processes in biological and

\footnotetext{
a) Author to whom correspondence should be addressed. Electronic mail: john.fulton@pnnl.gov
}

geochemical systems. For these reasons, it is essential to provide an accurate description of this system.

Current state-of-the-art simulation methods are based upon $a b$ initio approaches such as density functional theory (DFT)-based molecular dynamics (MD) simulations. Although DFT-MD methods are far more computationally intensive than MD methods using classical intermolecular potentials, DFT-MD offers the ability to simulate the complex, multicomponent systems that are common to many aqueous systems. While DFT methods have provided quantitative structural information for divalent cations ${ }^{10}$ and monovalent anions, ${ }^{11,12}$ there appears to be an, as yet, unresolved issue with monovalent cations. ${ }^{1,3,4,13}$ This is partially related to recent DFT improvements to treat long-range dispersion or van der Waals interactions. ${ }^{1,4,14}$

$\mathrm{X}$-ray (XRD) and neutron (ND) diffraction are the primary methods used to measure the $\mathrm{Na}-\mathrm{O}$ pair distribution functions. Aqueous $\mathrm{Na}^{+}$is particularly challenging since the neutron scattering length for $\mathrm{Na}(3.6 \mathrm{fm})$ is not largely different from the $\mathrm{O}$ of water $(5.8 \mathrm{fm})$ while a similar difficulty exists for XRD with $\mathrm{Na}(31.0 \mathrm{fm})$ and $\mathrm{O}(22.6 \mathrm{fm})$. A further complication is the necessity to deconvolute the measured $\mathrm{Na}-\mathrm{O}$ distribution (at $\sim 2.4 \AA$ ) from the partially overlapping O-O distribution (at $2.7 \AA$ ). With ND, there are added difficulties with nearby $\mathrm{O}-\mathrm{H}$ and anion-water distributions. Finally the presence of the salt can perturb the overall structure of water $(\mathrm{O}-\mathrm{O}$ distribution). Thus there is need for the best-possible 
scattering measurements with the highest possible spatial resolution and sensitivity.

State-of-the art x-ray and neutron sources and the associated scattering spectrometers are providing unprecedented insights into this very challenging problem. In this report, we measure the aqueous $\mathrm{Na}^{+}$structure using an XRD spectrometer and data analysis methods that have recently produced a much improved measurement of pure water. ${ }^{15,16}$ A key feature is that the high-Q measurements provide the full spatial resolution needed to fully resolve all features in the experimental pair distribution functions. We also note a recent ND study ${ }^{8}$ using the Empirical Potential Structure Refinement (EPSR) method with classical potentials that reported $\mathrm{Na}-\mathrm{O}$ distances of $2.34 \pm 0.14 \AA$ and $\mathrm{Na}-\mathrm{O}$ coordination number of $4.5 \pm 1.4$ at $5.5 \mathrm{~m} \mathrm{NaCl}$. A more direct method of ND scattering evaluation ${ }^{9}$ involves removal of the water-water contribution by subtraction of a null mixture ( $\mathrm{H}$ and $\mathrm{D})$ of pure water. This method gives a $\mathrm{Na}-\mathrm{O}$ distance of $2.34 \AA$ and a coordination number of $5.0 \pm 0.5$.

$\mathrm{X}$-ray absorption fine structure spectroscopy (XAFS) is another method that effectively probes these length scales, and in particular, the extended-XAFS (EXAFS) region is important since it contains the photoelectron backscattering processes that are due to spatial distribution of atoms in the sample. While EXAFS has been used to measure ion hydration structure with the same spatial resolution as XRD and ND, it is rarely been applied to light element ions ${ }^{17-20}$ since the low K edge energies create challenges for transmission through solutions and $\mathrm{x}$-ray windows. In this study, we use the latest generation of low energy beamlines to acquire the first EXAFS spectra for aqueous $\mathrm{Na}^{+}$. The advantage of EXAFS ${ }^{17}$ is the ability to isolate the signal from the $\mathrm{Na}-\mathrm{O}$ or $\mathrm{Na}-\mathrm{Cl}$ in the first solvent shell about the $\mathrm{Na}^{+}$in a way that the signal is completely free from interferences of the other pair-distribution functions in the sample (especially, $\mathrm{O}-\mathrm{O}$ ).

Molecular dynamics is a powerful tool to investigate the microscopic details of the solvation structure of ions in water. Both ab initio and classical simulations have been carried out in detail to study the structure of water ${ }^{21-24}$ and the perturbation due to the presence of salt. ${ }^{2,3,25-27,}$ In case of $a b$ initio simulations, the quality of the calculations depends on the choice of the exchange-correlation potential. However, the available DFT functionals are not able to describe the dispersion interactions satisfactorily. ${ }^{28,29}$ Various ways of including dispersion corrections along with commonly used functionals have been proposed. The structure of liquid water and aqueous $\mathrm{NaCl}$ solution have been studied by using the popular BLYP ${ }^{30,31}$ and $\mathrm{PBE}^{32}$ functionals along with Grimme D2 dispersion correction. ${ }^{33}$ However, neither the DFT nor DFT-D2 methods using these two functionals satisfactorily reproduce the water density. The use of a hybrid functional, e.g. PBE0 ${ }^{34}$ instead of $\mathrm{PBE}$, was found to soften the oxygen-oxygen radial distribution function for ambient water; ${ }^{35}$ however, it did not improve the $\mathrm{Na}-\mathrm{O}$ radial distribution function. ${ }^{2}$ On the contrary, the hybrid functional was found to produce lower numbers of hydrogen bonds per water molecule in the dilute $\mathrm{NaCl}$ solution. ${ }^{2}$ Bankura et al. ${ }^{3,4}$ has studied the solvation structure of $\mathrm{Na}^{+}$in water by using BLYP, PBE, and HCTH functionals along with Grimme D2 dispersion correction. To investigate the role of dispersion and other long range interactions, Soniat et al. ${ }^{1}$ recently studied the binding free energies of $\mathrm{Na}^{+}$in small water clusters using hybrid, dispersion corrected and range separated GGA DFT functionals. This study found that both dispersion correction and range separation together produced the best binding energy in comparison to high level $a b$ initio data.

Recently, we have applied the revPBE functional ${ }^{36}$ with the Grimme D3 dispersion correction ${ }^{37}$ to investigate the structure of water under ambient conditions and under pressure. ${ }^{16}$ This protocol reproduced the experimental radial distribution function satisfactorily. In light of that simulation, we have applied the same protocol to simulate $\mathrm{NaCl}$ in water system. Besides revPBE-D3, we also used the well-studied BLYP functional $^{30,31}$ with the D2 dispersion correction ${ }^{33}$ and compared those with revPBE-D3 data. Since $a b$ initio simulations are computationally expensive for larger systems at high salt concentrations, we used a classical simulation employing a $\mathrm{Na}-\mathrm{O}$ Lennard-Jones potential developed by Joung and Cheatham $(\mathrm{JC})^{38}$ for describing the ion-water interaction and SPC/E model $^{39}$ to describe the water-water interaction.

Our two objectives in this paper are to report significantly more accurate experimental measurements of the $\mathrm{Na}-\mathrm{O}$ structure using XRD and EXAFS and to simulate the solvation structure of $\mathrm{Na}^{+}$in water using comprehensive first-principles and classical molecular dynamics approaches and compare the theoretical predictions with experiment.

\section{METHODS AND THEORY}

\section{A. X-ray absorption fine structure experiments}

The Na K-edge (1070.8 eV) XAFS experiments were carried out at the Phoenix II, elliptical undulator beamline at the Swiss Light Source (SLS) at the Paul Scherrer Institute (PSI), Switzerland. The PHOENIX II endstation is located at the exit port of the X-Treme beamline. ${ }^{40}$ Briefly, an elliptical undulator serves as photon source, and a planar grating monochromator is used to generate monochromatic light. Sufficient rejection of high harmonics is achieved by appropriate choice of the cff values of the monochromator. Calibration of the monochromator was achieved by setting the inflection point of an $\mathrm{Al}$ foil spectrum to $1559.6 \mathrm{eV}$. In addition, the core-hole lifetime broadening for sodium is expected to be about $0.30 \mathrm{eV}$. The unfocused $1.0 \times 1.0 \mathrm{~mm}$ beam had a flux of approximately $10^{11}$ to $10^{12}$ photons/s. The sample chamber pressure was maintained at approximately $2.5 \times 10^{-4}$ mbar. Measurements were performed in fluorescence mode. The $\mathrm{X}$-ray fluorescence was detected using a 4-element Si-drift diode detector (manufacturer: Vortex). The current of the incoming beam $\left(I_{0}\right)$ was measured using the total electron yield signal from a $0.5 \mu$ s thin polyester foil, which was coated with $50 \mathrm{~nm}$ of nickel. The beam passed through this foil about $1 \mathrm{~m}$ upstream of the sample in a vacuum chamber held at about $10^{-6}$ mbar.

For the liquid samples, a membrane-window cell was used that is similar to what has been previously described ${ }^{20}$ that incorporated a $200 \mathrm{~nm}$ thick $\mathrm{Si}_{3} \mathrm{~N}_{4}$. Liquid transfer lines extended outside of the vacuum chamber for ease of sample introduction. $\mathrm{NaCl}$ (Sodium chloride (99.999\%) and 
$\mathrm{Na}\left(\mathrm{ClO}_{4}\right)$ (sodium perchlorate, 99.99\%) were obtained from Sigma-Aldrich and were used without further processing. The measurement temperature was $25^{\circ} \mathrm{C}$.

We used portions of the Athena and Artemis programs for the analysis of XAFS data ${ }^{41-43}$ while the theoretical standards were calculated using FEFF9. ${ }^{44,45}$ Signal from the atomic background function was removed from the backscattering signal, $\chi(k)$, by setting a Fourier filter distance, $\mathrm{R}_{\mathrm{bkg}}$, to 1.4 $\AA$ A . During fitting, the $k$-edge XAFS data were weighted by $k^{2}$ and windowed between $1.5<k<9.0 \AA^{-1}$ using a Hanning window with $d k=1.0 \AA^{-1}$. The fits were to both the real and imaginary parts of $\tilde{\chi}(R)$ in the region of $1.0<R<5.0 \AA$.

In the present study, the reported value of the coordination number from XAFS was adopted by first establishing the value of the core hole factor, $S_{0}^{2}=0.87$, through fitting to the $\mathrm{NaCl}$ crystalline standard. Estimation of the $\mathrm{Na}-\mathrm{O}$ coordination number and the disorder is an especially challenging task for a low- $Z$ atom since the fluorescence self-absorption correction is more difficult to apply quantitatively at these low energies. On the other hand, the $\mathrm{Na}-\mathrm{O}$ distance is only weakly correlated with amplitudes and hence the measured distance is quite accurate. $S_{0}^{2}$ has an associated uncertainty of about $25 \%$ that results in an approximate $\pm 25 \%$ uncertainty in the reported coordination number.

The atomic background function, $\mu_{0}(E)$, also contains two multi-electron absorption edges that are not fully removed through Fourier filtering. These features appear in the $\chi(k)$ data at $k=3.9$ and $5.1 \AA^{-1}$ and they correspond to the $K L_{\mathrm{II}, \mathrm{III}}$, and $K L_{\text {I }}$ transitions, respectively, that are similar to those observed for aqueous $\mathrm{Al}^{3+} .^{20}$ While the $K L_{\text {II,III }}$ transition is relatively strong and easy to identify, the $K L_{\mathrm{I}}$ transition is much weaker and the relative edge height is difficult to discern. Although the $k$ range is relatively short, the $\mathrm{Na}-\mathrm{O}$ distance is well resolved.

\section{B. High energy $x$-ray diffraction experiments}

The high energy $x$-ray diffraction measurements were made using the beamline 11-ID-C at the Advanced Photon Source (Argonne National Laboratory, USA). Monochromatic $\mathrm{x}$-rays with an incident energy of 114.76(1) $\mathrm{keV}$ illuminated the samples placed in thin-walled polyimide tubes. The $\mathrm{x}$-rays were detected with an amorphous silicon area detector (Perkin Elmer XRD1621). Scattering patterns were radially integrated using the fit $2 \mathrm{D}$ package ${ }^{46}$ while other corrections were applied using custom software. The measurements were made at room temperature.

\section{Molecular dynamics simulations}

\section{First principles simulations}

First principles molecular dynamics simulations were carried out in the canonical ensemble (NVT ensemble) using the Quickstep module of the CP2K software ${ }^{47}$ and periodic boundary conditions. A periodic cubic simulation box of side $14.23 \AA$ containing one $\mathrm{NaCl}$ ion pair solvated with 95 water molecules was used providing a $0.58 \mathrm{~m}$ solution and producing a density of 0.0333 molecules per $\AA^{3}\left(1.02 \mathrm{~g} / \mathrm{cm}^{3}\right)$. The $\mathrm{Na}^{+}$and $\mathrm{Cl}^{-}$ were initially placed in the simulation box by replacing two water molecules at a distance of $7 \AA$ from each other. We maintained a temperature of $300 \mathrm{~K}$ using the Nośe-Hoover chain thermostat ${ }^{48}$ having the characteristic frequency of $3000 \mathrm{~cm}^{-1}$. The valence electrons were treated explicitly at the DFT level employing both revPBE ${ }^{36}$ and BLYP $^{30,31}$ functionals and dual basis sets with both TZV2P or DZVPMOLOPT-SR (Gaussian type orbital) with density from a cutoff of $400 \mathrm{Ry}{ }^{47}$ The core electrons on all atoms were represented by Goedecker-Teter-Hutter (GTH) pseudopotentials. ${ }^{49}$ To investigate the role of dispersion in describing the structure of solvated $\mathrm{Na}^{+}$ion, we used the Grimme D3 dispersion correction $^{37}$ for the revPBE and Grimme D2 dispersion correction $^{33}$ for the BLYP functional within the DFT protocol. As stated earlier, the choice of revPBE-D3 protocol for the simulations of $\mathrm{Na}^{+}$ion in water was motivated by a recent successful study of ambient and high-pressure water in our group. ${ }^{16}$ A time step of 0.5 fs was used to generate a $80 \mathrm{ps}$ trajectory. The data were collected from the last $50 \mathrm{ps}$ of that trajectory.

\section{Classical simulations}

Classical simulations were carried out using the JC force field ${ }^{38}$ and SPC/E water model. ${ }^{39}$ In the JC force field, the ion water interaction parameters were determined by fitting both the experimental ion hydration free energies and crystal properties. Since this model included crystal properties along with hydration free energies, it was suitable to describe the ion-ion interactions in high concentration salt solution. ${ }^{50}$ For the dilute solution, the simulation box contained one $\mathrm{NaCl}$ ion pair and 2625 water molecules in a cubic box of $42.76 \AA$ in each side. The $\mathrm{Na}^{+}$and $\mathrm{Cl}^{-}$were initially placed in the simulation box by replacing two water molecules at a distance of $15 \AA$ from each other. For the $6 \mathrm{~m}$ solution, the simulation box contained $300 \mathrm{NaCl}$ ion pair and 2350 water molecules in a cubic box of $43.59 \AA$ in each side. The simulations were performed in a constant volume ensemble at a temperature of $300 \mathrm{~K}$. Electrostatics were carried out using the smooth version of the particle mesh Ewald (SPME) method ${ }^{51}$ with a real space cutoff of $7.5 \AA$. A time step of 0.5 fs was used. The simulations were carried out for $2 \mathrm{~ns}$ of equilibration and then a period of 4 ns of production.

\section{Calculating the potential of mean force (PMF)}

We used the umbrella sampling technique for calculating the potential of mean force (PMF) as a function of the number of coordinated water molecules in the first solvation shell of $\mathrm{Na}^{+}$ion. Two different systems were prepared having different box sizes to explore the effect of system size on the free energy profile. The first system was comprised of a single $\mathrm{Na}^{+}$solvated by 64 water molecules, in a periodic cubic box of length $12.56 \AA$ producing a density of $1.03 \mathrm{gm} / \mathrm{cc}$. The second simulation was a larger system containing one $\mathrm{Na}^{+}$ion and 96 water molecules in a periodic cubic box of length $14.3 \AA$ producing a density of $1.03 \mathrm{gm} / \mathrm{cc}$. We also prepared another system to check the effect of density, which had a single $\mathrm{Na}^{+}$solvated by 64 water molecules but the density was maintained to be $0.997 \mathrm{gm} / \mathrm{cc}$.

The coordination number $(n)$ was computed using the same functional form for as in a previous study, ${ }^{27}$ 


$$
n=\sum_{i}^{N o} \frac{1-\left(\frac{r_{i}}{r_{0}}\right)^{p}}{1-\left(\frac{r_{i}}{r_{0}}\right)^{q}},
$$

where $p$ and $q$ are integers, usually $p=16$ and $q=32, r_{i}$ is the distance between the $\mathrm{Na}^{+}$and oxygen $i$, and $r_{0}=3.0 \AA$. The free energy was computed using the umbrella sampling technique. Sampling windows for $n$ ranged from 4.9 to 8.1 and were equally spaced by 0.1 apart employing harmonic umbrella potentials of the from $\mathrm{V}(\mathrm{n})=\mathrm{k}\left(\mathrm{n}_{0}-\mathrm{n}\right)^{2}$ with a force constant $\mathrm{k}$ of $956 \mathrm{kcal} \mathrm{mol}^{-1}$. In each umbrella window, a trajectory of at least $50 \mathrm{ps}$ was collected after $5 \mathrm{ps}$ of equilibration. The weighted histogram analysis method (WHAM) was employed to extract a free energy profile from these histograms.

\section{Calculating the total $x$-ray pair distribution function, $G_{x}(r)$}

The total X-ray pair distribution function $G_{x}(r)$ was calculated using the following equation:

$$
G_{X}(r)=\frac{1}{(2 \pi)^{3} \rho} \int_{0}^{\infty} 4 \pi Q^{2} F^{x}(Q) \frac{\sin (Q r)}{Q r} d Q,
$$

where $\mathrm{F}^{\mathrm{x}}(\mathrm{Q})$ is the $\mathrm{X}$-ray weighted total-scattering structure factor as shown in the following equation:

$$
F^{x}(Q)=\rho \sum_{i}^{n} c_{i} c_{j} f_{i j}(Q) \int_{0}^{\infty} 4 \pi r^{2}\left[g_{i j}(r)-1\right] \frac{\sin (Q r)}{Q r} d r,
$$

where

$$
f_{i j}(Q)=f_{i}(Q) f_{j}(Q) /\left[\sum_{i}^{n} c_{i} f_{i}(Q)\right]^{2} .
$$

The X-ray scattering factor $f_{i}(Q)$ was analytically developed from Gaussian fits of the scattering factor obtained from atomic wavefunctions ${ }^{52}$ and $\mathrm{g}_{\mathrm{ij}}(\mathrm{r})$ are the atom-atom radial distribution functions calculated from the simulated trajectories.

\section{Simulating the K-edge XANES spectra}

XANES calculations were carried out at the Na K-edge for $\mathrm{Na}^{+}$solvated by water molecules using the restricted excitation window (REW) TDDFT approach ${ }^{53}$ as implemented in the NWChem program. ${ }^{54}$ A set of 50 snapshots was taken from the $a b$ initio molecular dynamics simulations for the calculation. We used clusters with one $\mathrm{Na}^{+}$solvated by 40 water molecules and implicit COSMO dielectric continuum. In the XANES calculations, the Sapporo-QZP-2012 all electron basis set ${ }^{55}$ was used for the single $\mathrm{Na}^{+}$ion while the $6-311 \mathrm{G}^{* *}$ basis $\operatorname{set}^{56}$ was used for the surrounding water $\mathrm{O}$ and $\mathrm{H}$ atoms. The exchange correlation was treated with the BHLYP functional. ${ }^{57}$ The spectra were Lorentzian broadened by $1 \mathrm{eV}$ to compare with the experiments.

\section{RESULTS AND DISCUSSION}

\section{A. Aqueous $\mathrm{Na}^{+}$from molecular dynamics simulations}

To investigate the local solvation environment around the $\mathrm{Na}^{+}$ion, we calculated the $\mathrm{Na}-\mathrm{O}$ radial distribution func- tions and the corresponding running coordination numbers for different $a b$ initio and classical simulations as shown in Figure 1. A summary of the numerical values for the position of the first maximum and the coordination numbers is given in Table I.

In all levels of theories, we found that the first solvation shell is characterized by a sharp first peak and a deep but nonzero first minimum. The average $\mathrm{Na}-\mathrm{O}$ distance for the first solvation shell is 2.45 and $2.46 \AA$ for both of the TZV2P and MOLOPT basis sets, respectively. These values are slightly overestimated when compared to our experimentally derived values (reported below) of $2.37 \AA$ (from EXAFS) and $2.38 \AA$ (from XRD). When the dispersion corrections are applied in the simulations, the $\mathrm{Na}-\mathrm{O}$ distances are increased by $0.08-0.10 \AA$, which significantly worsens the agreement compared with experiment. The increase in the Na-O distance in presence of the dispersion corrections with revPBE functional is consistent with the previous computational studies by others that use popular functionals and D2 Grimme dispersion corrections (BLYP/BLYP-D2, PBE/PBE-D2, and $\mathrm{HCTH} / \mathrm{HCTH}-\mathrm{D} 2){ }^{3}$ Even though the $\mathrm{Na}-\mathrm{O}$ distance without dispersion is closer to the experimental value, it is well known that the density of the water is underestimated in absence of the dispersion correction. ${ }^{58}$ To check the density of our simulation box, we ran constant pressure simulations with the same box size and periodic boundary conditions, which provided a density of $1.07 \pm 0.02 \mathrm{~g} / \mathrm{cc}$ and $0.80 \pm 0.03 \mathrm{~g} / \mathrm{cc}$ for the simulations with and without the dispersion correction, respectively (the experimental density for $0.5 \mathrm{M} \mathrm{NaCl}$ solution is $1.02 \mathrm{~g} / \mathrm{cc}$ ). We also checked whether the discrepancy of the calculated $\mathrm{Na}-\mathrm{O}$ distance is an artifact due to the choice of the pseudopotentials, so we compared these results with results using the all-electron GAPW approach ${ }^{59}$ in CP2K. The $\mathrm{Na}-\mathrm{O}$ distance found for the GAPW calculation was $2.51 \AA$ which is quite similar to the calculated distance of $2.53 \AA$ through the use of pseudopotentials. Thus the choice of pseudopotentials does not significantly alter the $\mathrm{Na}-\mathrm{O}$ distances.

The height and the shape of the first peak with and without dispersion corrections are also found to be different. With both the basis sets, we found that the height of the first peak decreased with dispersion correction by 0.5 (from 5.2 to 4.7 ) and by 1.0 (from 5.1 to 4.1) for TZV2P and DZVP-MOLOPTSR, respectively. However, for DZVP-MOLOPT-SR basis set, the first peak became slightly broader and the peak height decreased more than for the TZV2P basis set. In the absence of dispersion correction, both the TZV2P and MOLOPT basis sets were producing similar radial distribution function. However, in presence of the dispersion correction, the difference became more pronounced. This is consistent with our previous observations for the simulation of liquid bulk water, that this difference is due to the D-3 dispersion correction, which was originally optimized with TZV2P basis set. With inclusion of the dispersion correction, the $\mathrm{Na}-\mathrm{O}$ coordination number for the first solvation shell (integrated up to $3.4 \AA$ cutoff) increased to the value of 6.0 and 6.1 for TZV2P and MOLOPT basis set, respectively. The increase in the coordination number reported here when $\mathrm{D} 3$ dispersion correction was employed with revPBE functional is consistent with the 

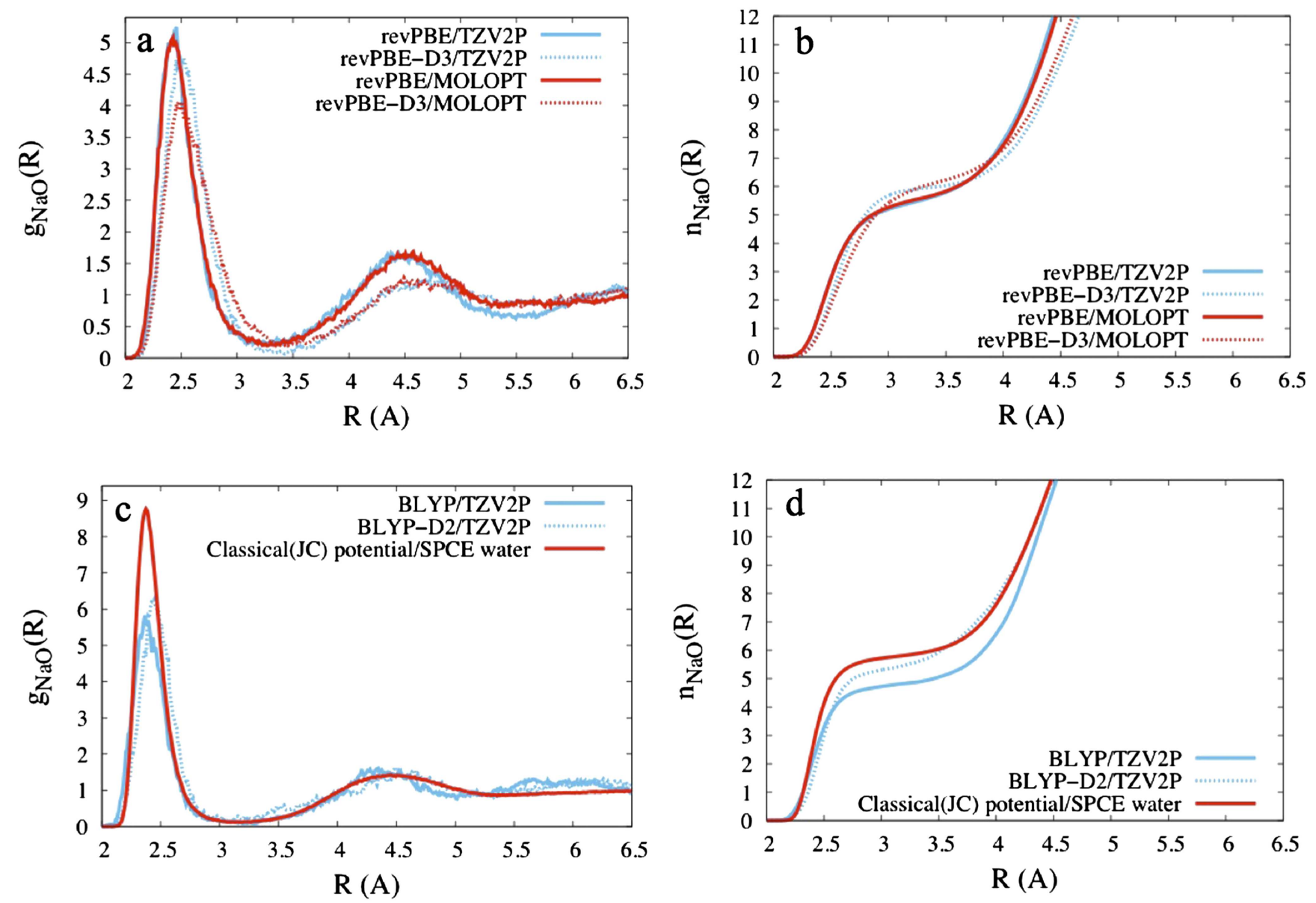

FIG. 1. Comparison of simulated Na-O radial distribution functions ((a) and (c)) and the corresponding running coordination numbers ((b) and (d)). Top: revPBE and revPBE-D3 level of theory using both TZV2P and DZVP-MOLOPT-SR basis sets. Bottom: BLYP and BLYP-D2 level of theory using TZV2P basis set, and classical JC potential with SPC/E water model.

previous calculations where D2 dispersion correction was found to increase the coordination number by about $0.5-0.9$ for BLYP, PBE, and HCTH functionals. ${ }^{3}$ In contrast to the D2 dispersion correction where the height of the first peak was found to increase, ${ }^{3}$ we found that in case of D3, the height of the first peak decreased. The coordination number of $5.7 \mathrm{cal}-$ culated from revPBE level is again in better agreement with the experimental value of $5.5 \pm 0.3$ estimated in this XRD study

TABLE I. The position of the average Na-O distance $\left(r_{\mathrm{Na}-\mathrm{O}}\right)$ in the RDF and the coordination number $\left(\mathrm{n}_{\mathrm{Na}-\mathrm{O}}\right.$ ) for the first solvation shell (up to $3.4 \AA)$.

\begin{tabular}{lccc}
\hline \hline & \multicolumn{3}{c}{ Structure } \\
\cline { 2 - 4 } System & 2.45 & 24.2 & 5.7 \\
\hline rev-PBE (TZV2P) & 2.53 & 31.9 & 6.0 \\
rev-PBE-D3 (TZV2P) & 2.46 & 25.7 & 5.7 \\
rev-PBE (DZVP-MOLOPT-SR) & $\AA)^{\mathrm{a}}$ & $\sigma^{2} \times 10^{3}\left(\AA^{2}\right)^{\mathrm{b}}$ & $\mathrm{CN}_{\mathrm{Na}-\mathrm{O}}$ \\
rev-PBE-D3 (DZVP-MOLOPT-SR) & 2.56 & 44.5 & 6.1 \\
Classical (JC potential) & 2.39 & 11.5 & 5.5 \\
BLYP & 2.40 & 18.0 & 4.9 \\
BLYP-D2 & 2.46 & 17.9 & 5.7 \\
\hline \hline
\end{tabular}

${ }^{a}$ Values derived from the centroid of a fitted Gaussian distribution.

${ }^{\mathrm{b}} \sigma^{2}$ is the mean square variation in the distance obtained from the full width at half maximum of the Gaussian fit. compared to the coordination number of 6.0 calculated from revPBE-D3 level.

The $\mathrm{Na}-\mathrm{O}$ distance obtained from the radial distribution function calculated from the classical simulation using JC force field with SPC/E water is $2.39 \AA$. The corresponding coordination number is 5.5. Interestingly, this $\mathrm{Na}-\mathrm{O}$ distance of $2.39 \AA$ from classical simulation is the closest to the experimental value (see Table II). The Na-O distance of $2.34 \AA$ obtained previously by using the Dang polarizable model ${ }^{60}$ was also close to the experimental value. It appears that classical potentials $\left(\mathrm{JC}^{38}\right.$ and Dang $\left.{ }^{60}\right)$, that have been parameterized to predict sets of experimental properties, are in general closer to the experimental values.

The discrepancy in producing the ion-water distance and the coordination number of waters around the ion through DFT simulations might be due to the limitation of the functional used in our simulations. It is critical to get the correct choice of the functional that will concurrently reproduce both the ionwater and the water-water interactions. revPBE-D3 was found to produce water-water interactions satisfactorily; however, it is overestimating both the distance and the coordination number in case of ion-water. Previous simulations showed that PBE and PW91 functionals produce a coordination number of $\sim 5,{ }^{61-64}$ however, using PBE without dispersion correction produces over-structured water. ${ }^{65}$ The ion-water distances 

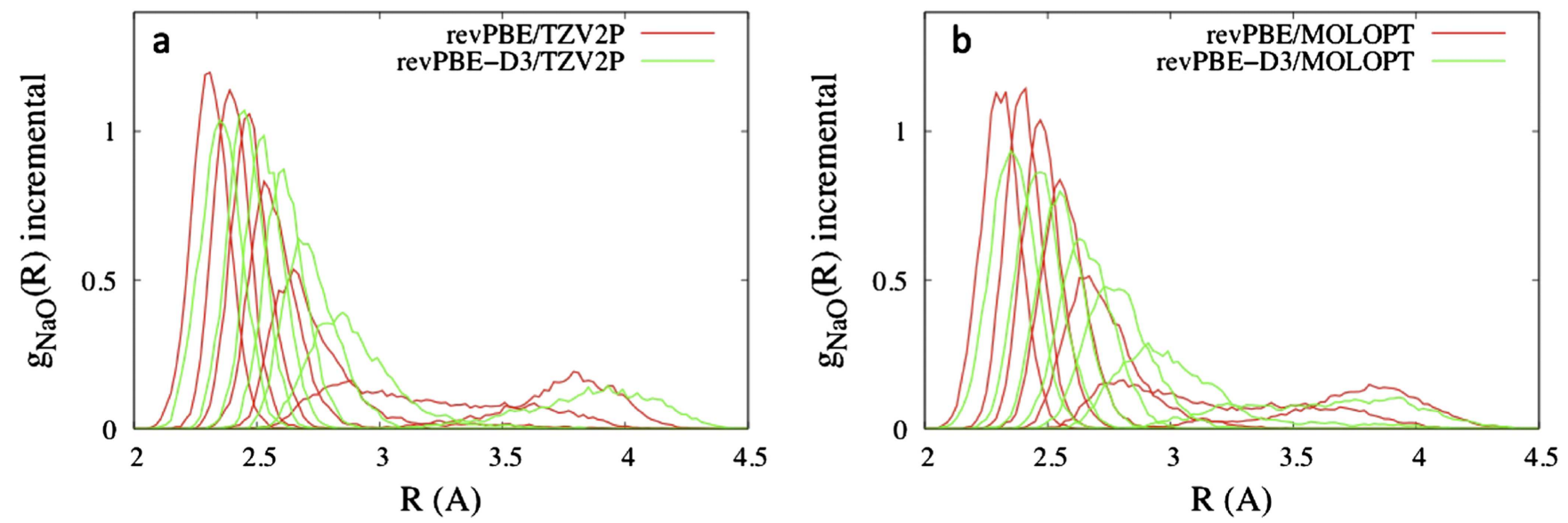

FIG. 2. Incremental distribution function, $\mathrm{g}_{\mathrm{NaO}}(\mathrm{r})$, for (a) revPBE/TZV2P level of theory and (b) revPBE/molopt level of theory with and without dispersion corrections (D3).

reported from PBE simulations are conflicting and cover a range of distances (2.37-2.49 $\AA^{2} .^{2,3,61}$ The lack of consistency in reported distances may be due to differences in simulation protocol including the simulation temperatures, namely, $300 \mathrm{~K}$ versus an elevated temperature of $+30 \mathrm{~K}$ that has been used to mimic quantum effects. The addition of dispersion correction to the PBE functional was also found to worsen the agreement to the experiment. ${ }^{3}$ The limitations of current functionals employed and discussed here indicate a need to develop new functionals that produce accurate ion hydration structure.

More insight into the role of dispersion on the solvation structure is evident by inspecting the incremental radial distribution functions for the closest seven waters around $\mathrm{Na}^{+}$ion as shown in Figure 2. For the revPBE/TZV2P simulations, the first 5 water molecules remained within the first-shell distance of $3.2 \AA$ from the central $\mathrm{Na}^{+}$ion while the 6th water showed a broad distribution between 2.5 and $4.0 \AA$. The movement of this 6th water between the first and second solvation shell is consistent with the coordination number that lies between 5 and 6. When D3 correction was applied, the first 5 water molecules moved to a slightly larger distance and broader distribution, while the 6th water molecule showed the most significant change. Its distribution became narrower within 2.5 to $3.4 \AA$ region which contributed to the increase in the coordination number of the first solvation shell. The 7th water molecule was not found to contribute in the population of the first solvation shell in either case. Unlike the TZV2P basis set, the revPBE-D3/DZVP-MOLOPT-SR simulations showed that both 6th and 7th water molecules spent more time within the first solvation shell and contributed to the population increase.

To understand the free energy profile in the coordination space for the $\mathrm{Na}^{+}$at various level of theories, we have calculated the PMFs as shown in Figure 3. For uncorrected BLYP level of theory, the six-coordinated state is $\sim 2 \mathrm{kcal} / \mathrm{mol}$ higher in energy than the five-coordinated state. However, when the dispersion correction is applied, the most stable well moves to six-coordinated state. Both five and seven coordinated states are slightly below a $2 \mathrm{kcal} / \mathrm{mol}$ energy barrier. For revPBE-D3, the most stable well is also the six-coordinated one. However, in contrast to BLYP-D2, there is no local minimum for sevencoordinated species. The results of revPBE-D3 free energies for different simulation cell sizes are the same within the error bar. Therefore, dispersion corrected DFT methods (BLYP-D2 and revPBE-D3) provide the six-coordinated species as the most stable one.

We also focused on the resulting population densities at different local minima as shown in Figure 4. BLYP level of theory does not show any significant population at the sixcoordinated state. At the BLYP-D2 level, $85 \%$ of the $\mathrm{Na}^{+}$ ions remain populated by six water molecules and the remaining $15 \%$ by between five and six water molecules. In case of

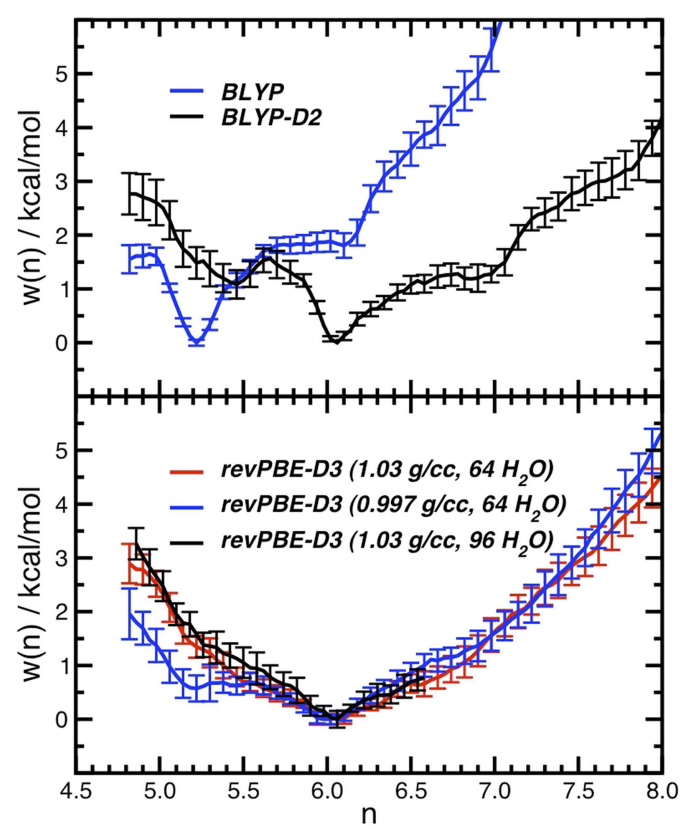

FIG. 3. Free-energy versus the $\mathrm{Na}^{+}$-oxygen coordination number (n) in aqueous solution obtained from umbrella sampling for (upper panel) BLYP (blue), BLYP-D (black) for the small system containing 64 water molecules and (lower panel) revPBE-D3 (blue) and revPBE-D3 (red) for the small system containing 64 water molecules with fixed density of 0.997 and $1.03 \mathrm{~g} / \mathrm{cc}$, respectively. In black, the free-energy for revPBE-D3 is shown for the larger system containing 96 water molecules at fixed density of $1.03 \mathrm{~g} / \mathrm{cc}$. 


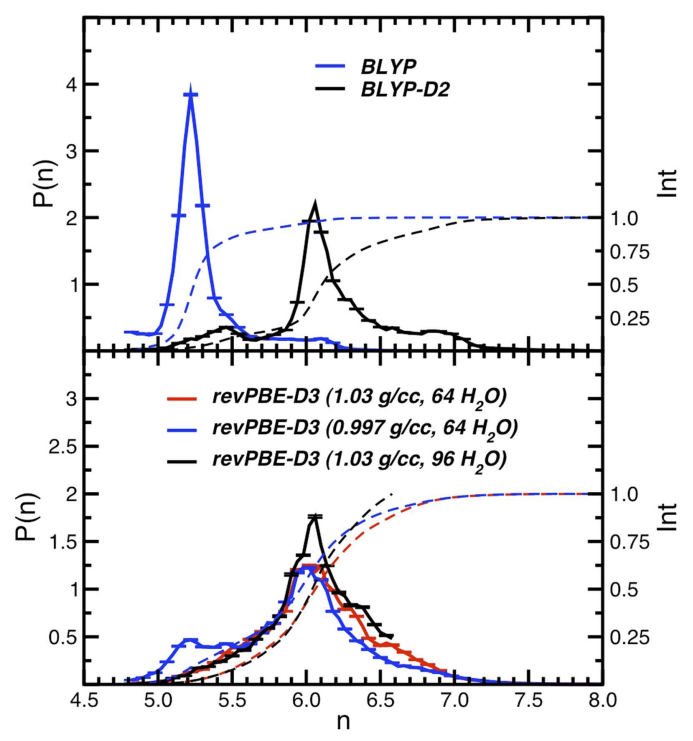

FIG. 4. Normalized probability distribution of the $\mathrm{Na}^{+}$-oxygen coordination number (n) in aqueous solution obtained from umbrella sampling for (upper panel) BLYP (blue), BLYP-D (black) for the small system containing 64 water molecules and (lower panel) revPBE-D3 (blue) and revPBE-D3 (red) for the small system containing 64 water molecules with fixed water density of 0.997 and $1.03 \mathrm{~g} / \mathrm{cc}$, respectively. In black, the probability distribution for revPBED3 is shown for the larger system containing 96 water molecules at fixed water density of $1.03 \mathrm{~g} / \mathrm{cc}$.

revPBE-D3, for both system sizes, the $\mathrm{Na}^{+}$ion always remains populated by six water molecules. Only in case of the system having a lower density $(0.997 \mathrm{~g} / \mathrm{cc})$ is there a $30 \%$ population of the five coordinate species and $70 \%$ population of the six coordinate species.

\section{B. Aqueous $\mathrm{Na}^{+}$structure from EXAFS experiment}

The experimental EXAFS spectra for three aqueous $\mathrm{Na}^{+}$ solutions are shown in Figure 5. The solutions include $6 \mathrm{~m}$ $\mathrm{NaCl}, 6 \mathrm{~m} \mathrm{Na}\left(\mathrm{ClO}_{4}\right)$, and $1 \mathrm{~m} \mathrm{NaCl}$ solutions from which the effect of the counterion $\left(\mathrm{Cl}^{-}\right.$or $\left.\mathrm{ClO}_{4}^{-}\right)$and the effect of concentration $(1$ and $6 \mathrm{~m} \mathrm{NaCl})$ on the first shell structure can be determined. For monovalent ions, the EXAFS signal primarily arises from photoelectron backscattering of the atoms within the first shell that are either the $\mathrm{O}$ atoms of water or atom(s) of the anion that would form a contact ion pair with the cation. The scattering signal from second and higher shells is negligible for monovalent cations since the disorder (the sets of scattering paths over this range of distances are out-of-phase) and distances are too high. One of the strengths of EXAFS is the ability to probe local structure about an ion of interest without the interfering contributions from the other pair distribution functions in the solution (especially $\mathrm{g}_{\mathrm{OO}}(\mathrm{r})$ and $\mathrm{g}_{\mathrm{ClO}}(\mathrm{r})$ ) as in XRD or ND methods). In the $k^{2}$-weighted $\chi(k)$ plot of Figure 5(a), there is a $K L_{\text {II,III }}$, multi-electron excitation feature at about $k$ $=3.9 \AA^{-1}$ and potentially a weaker feature at $5.1 \AA^{-1}$ due to the $K L_{\mathrm{I}}$ transition. These features are not expected to significantly distort the frequency information of the main oscillations in the $\chi(k)$ plot that are from scattering from O's of water in the first shell.
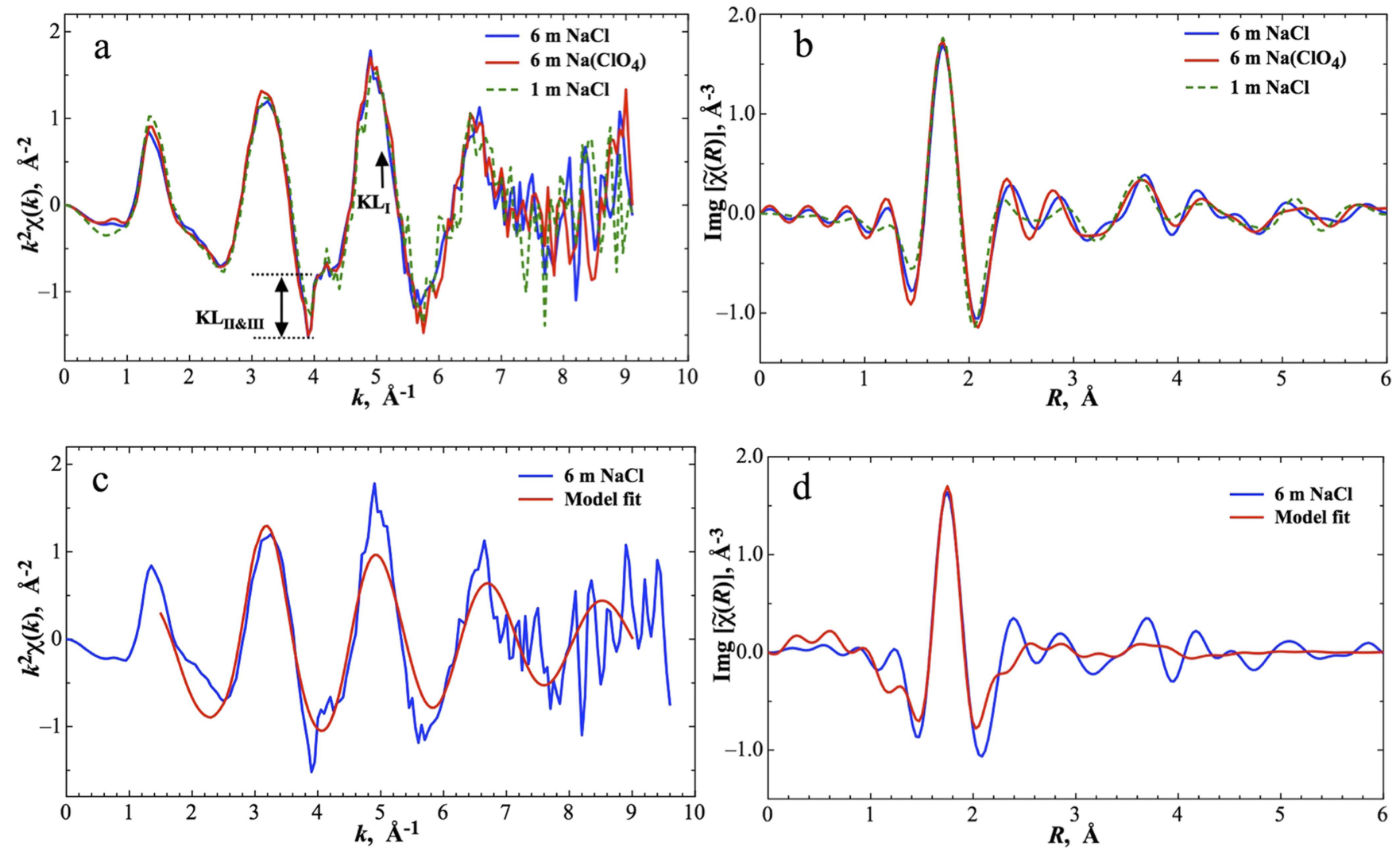

FIG. 5. (a) $\mathrm{Na}$ K-edge, $k^{2}$-weighted $\chi(k)$ plots for aqueous $6 m \mathrm{NaCl}, 6 m \mathrm{Na}\left(\mathrm{ClO}_{4}\right)$, and $1 \mathrm{~m} \mathrm{NaCl}$ solutions as indicated. (b) The corresponding $k^{2}$-weighted $\operatorname{Im}[\tilde{\chi}(R)]$ plots. (c) Na K-edge, $k^{2}$-weighted $\chi(k)$ plots for aqueous $6 \mathrm{~m} \mathrm{NaCl}$ solution. (d) The corresponding $k^{2}$-weighted Im $[\tilde{\chi}(R)]$ plots. The experimental data (blue lines) and the model fit (red lines) to the FEFF9 structural model are shown. The measurement temperature is $25^{\circ} \mathrm{C}$. 
TABLE II. Structural parameters from XAFS and XRD fits for aqueous $\mathrm{Na}^{+}$first-shell structure.

\begin{tabular}{lcccr}
\hline \hline & \multicolumn{4}{c}{ Structure } \\
\cline { 2 - 4 } System & $R_{\mathrm{O}}^{1 \text { st }}(\AA)$ & $\sigma^{2} \times 10^{3}\left(\AA^{2}\right)$ & $C_{3} \times 10^{3}\left(\AA^{3}\right)$ & $\mathrm{CN}_{\mathrm{Na}-\mathrm{O}}$ \\
\hline $\mathrm{Na}^{+} \operatorname{EXAFS}(6 \mathrm{~m} \mathrm{NaCl})$ & $2.372(024)$ & $6.1(3.0)$ & $5.2(2.4)^{\mathrm{a}}$ & $5.4(1.3)$ \\
$\mathrm{XRD}(6 \mathrm{~m} \mathrm{NaCl})$ & $2.384(003)$ & $20.6(1.0)^{\mathrm{b}}$ & $\cdots$ & $5.5(0.3)$ \\
$\mathrm{XRD}(2.5 \mathrm{~m} \mathrm{NaCl})$ & $2.381(005)$ & $20.0(2.0)^{\mathrm{b}}$ & $\cdots$ & $5.9(0.6)$ \\
\hline \hline & ${ }^{\mathrm{a}}$ Third cumulant or asymmetry parameter in the XAFS expansion. \\
${ }^{\mathrm{b}} \sigma^{2}$ is the mean square variation in the distance obtained from the full width at half maximum of the Gaussian fit.
\end{tabular}

Within the experimental uncertainties, the spectra for these three different solutions $\left(6 \mathrm{~m} \mathrm{NaCl}, 6 \mathrm{~m} \mathrm{Na}\left(\mathrm{ClO}_{4}\right)\right.$, and $1 \mathrm{~m} \mathrm{NaCl})$ are the same, both with respect to the $\chi(k)$ plot, but also in the Fourier transformed $\operatorname{Im}[\tilde{\chi}(R)]$ plots of Figure $5(b)$. The results thus indicate that there is little or no ion pairing with $\mathrm{Cl}^{-}$. While $\mathrm{Cl}^{-}$has the potential to form a contact ion pair with $\mathrm{Na}^{+}, \mathrm{ClO}_{4}^{-}$is considered to be a very weakly associating anion with much lower probability of contact ion pair with $\mathrm{Na}^{+}$. Formation of contact ion pair would be manifested in these spectra in two different ways. First, the entrance of the anion into the first shell would displace water molecules thereby decreasing the intensity of the $\mathrm{Na}-\mathrm{O}$ scattering peak at $1.9 \AA$ in Figure 5(b). The existence of $\mathrm{Na}-\mathrm{Cl}$ contact ion pair species would also lead to a new scattering peak at slightly longer distance of the $\mathrm{Na}-\mathrm{O}$. Neither one of these changes is observed in the experimental spectra thus suggesting that $\mathrm{Na}^{+}$ is primarily fully hydrated under these conditions. A fractional degree (less than $\sim 0.25$ ) of ion pairing probably would not be detected. In our classical simulation of $6 \mathrm{M} \mathrm{NaCl}$ solutions, there were $0.27 \mathrm{Cl}^{-}$per $\mathrm{Na}^{+}$ion at the ion pair position (within $3.5 \AA$ ) indicating $27 \%$ ion pairing as obtained from the $\mathrm{Na}-\mathrm{Cl}$ radial distribution function. In the dilute solution, $(1 \mathrm{NaCl}$ ion pair in 2625 water molecules), the $\mathrm{Cl}^{-}$never forms an ion pair within the simulation period as it was initially placed far apart from the $\mathrm{Na}^{+}$ion.

Figures 5(c) and 5(d) show the results of fitting the $6 \mathrm{~m}$ $\mathrm{NaCl}$ experimental data to the theoretical standards (FEFF9) while the resultant parameter from this fit is listed in Table II. The measured $\mathrm{Na}-\mathrm{O}$ bond distance is $2.37 \pm 0.02 \AA$ with a water coordination number about the $\mathrm{Na}^{+}$of $5.4 \pm 1.3$.
Figures 6(a) and 6(b) show the experimental and simulated K-edge XANES data of $\mathrm{Na}^{+}$in water, respectively. While the fluorescence self-absorption corrections used in the XANES amplitude normalizations can be challenging to treat quantitatively, the observed changes in the peak positions and shapes can be clearly ascribed to changes in the first and second shell structure. While the EXAFS region is primarily sensitive to the first-shell structure, the XANES region can have strong contributions from structure in the second and higher shells. Aziz et al. ${ }^{66}$ have reported on the effect of $\mathrm{NaCl}$ concentration on changes in the XANES structure. In that study, at high concentrations $(5 \mathrm{~m} \mathrm{NaCl})$, there is a $0.5 \mathrm{eV}$ shift in the "white line" peak at $1080 \mathrm{eV}$ to slightly lower energies and a new shoulder at $1075 \mathrm{eV}$ becomes more distinct. The same spectral changes are observed in this study as shown in Figure 6(a). Aziz et al. ${ }^{66}$ also showed, through an optimization of small $\mathrm{Na}^{+} / \mathrm{H}_{2} \mathrm{O} / \mathrm{Cl}^{-}$clusters, that these spectral changes are primarily due to formation of solvent-share ion pair at a $\mathrm{Na}-\mathrm{Cl}=3.9 \AA$. Such a structure is consistent with the EXAFS conclusions in Sec. III B indicating the lack of appreciable contact ion pairs $(\sim 2.8 \AA)$ in the $6 \mathrm{~m} \mathrm{NaCl}$ solution.

The two sets of simulated XANES spectra in Figure 6(b) were obtained by averaging over different ensembles from the DFT-MD trajectory, one having a $\mathrm{Na}^{+}$coordinated by an average of 5.5 water molecules and the other having $\mathrm{Na}^{+}$ coordinated by 6 water molecules. Each XANES spectrum represents an ensemble of 50 configurations containing one $\mathrm{Na}^{+}$solvated by the 40 nearest-neighbor water molecules with an implicit COSMO dielectric continuum. The set of
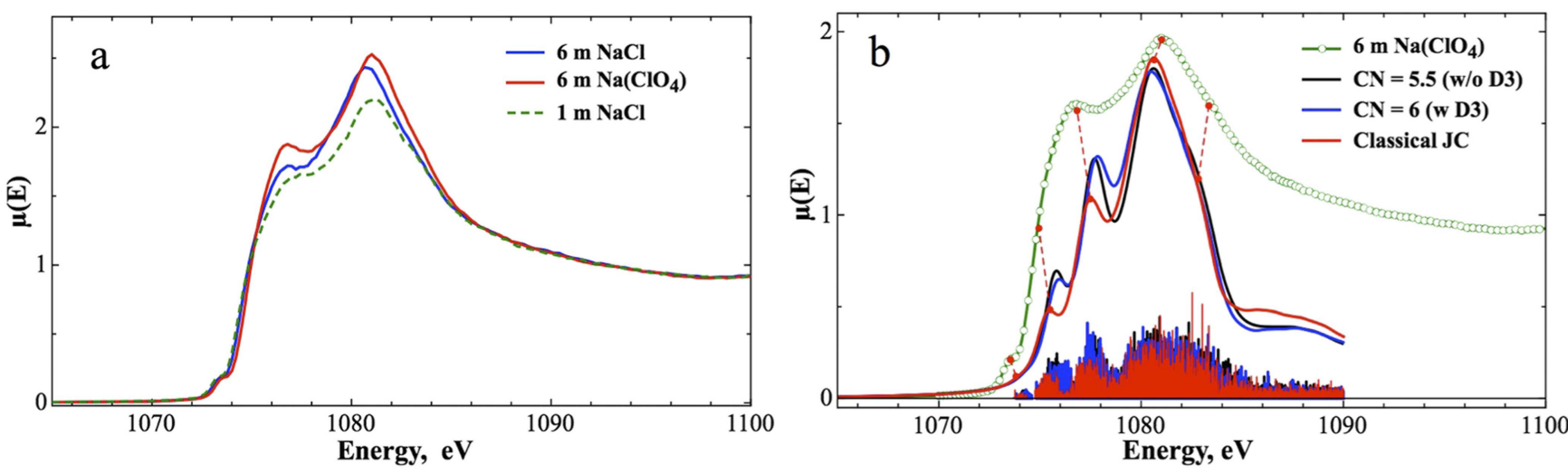

FIG. 6. (a) A series of normalized XANES spectra, $\mu(E)$, for aqueous $6 m \mathrm{NaCl}, 6 m \mathrm{Na}\left(\mathrm{ClO}_{4}\right)$, and $1 m \mathrm{NaCl}$ solutions as indicated. (b) Comparison of TDDFT XANES generated from an ensemble of MD trajectory snapshots with the experimental spectrum for aqueous $6 m \mathrm{Na}\left(\mathrm{ClO}_{4}\right)$. The measurement temperature is $25^{\circ} \mathrm{C}$. 
snapshots in which $\mathrm{Na}^{+}$is coordinated with 5.5 waters was harvested from the revPBE/TZV2P simulations without the dispersion corrections, while the 6-coordinated configurations were harvested from the revPBE-D3/TZV2P simulation. An overall shift of $+10.7 \mathrm{eV}$ was applied to the calculated XANES spectra. The peaks at 1073.5 and $1075 \mathrm{eV}$ are due primarily to $\mathrm{Na}(1 \mathrm{~s}) \rightarrow \mathrm{Na}(3 \mathrm{~s}, 3 \mathrm{p}) \mathrm{O}(3 \mathrm{~s}) \mathrm{H}(1 \mathrm{~s})$ or $\mathrm{Na}(3 \mathrm{~s}) \mathrm{O}(3 \mathrm{~s}) \mathrm{H}(1 \mathrm{~s})$ transitions while the peaks at 1076.5 and $1081 \mathrm{eV}$ are due primarily to $\mathrm{Na}(1 \mathrm{~s}) \rightarrow \mathrm{Na}(3 \mathrm{~s}, 3 \mathrm{p}) \mathrm{H}(1 \mathrm{~s})$ transitions. For both simulated cases, the predicted XANES peaks show a one-toone correspondence with features in the experimental spectra. There are only small differences between the spectra between the 5.5-coordinated and 6-coordinated ensembles primarily a small shift in the primary peak at $1080 \mathrm{eV}$. These results indicate that K-edge XANES is not particularly sensitive to the water coordination number in the $\mathrm{Na}^{+}$first shell.

\section{Aqueous $\mathrm{Na}^{+}$structure from XRD experiment}

Figure 7 shows the $x$-ray structure factors and pair distribution functions for pure water and the $2.5 \mathrm{~m}$ and $6.0 \mathrm{~m}$ $\mathrm{NaCl}$ solutions. The background removal and data treatment follows methods previously described. ${ }^{15,16}$ It should be noted that in the present analysis, the modified atomic normalization scheme is used, rather than the molecular scheme. This atomic scheme leaves in the intra-molecular water contributions to $S_{X}(Q)$ and $g_{X}(r)$, this is in contrast to many published $\mathrm{X}$-ray structure factors for water which use the molecular scheme which subtracts out intra-molecular structure from
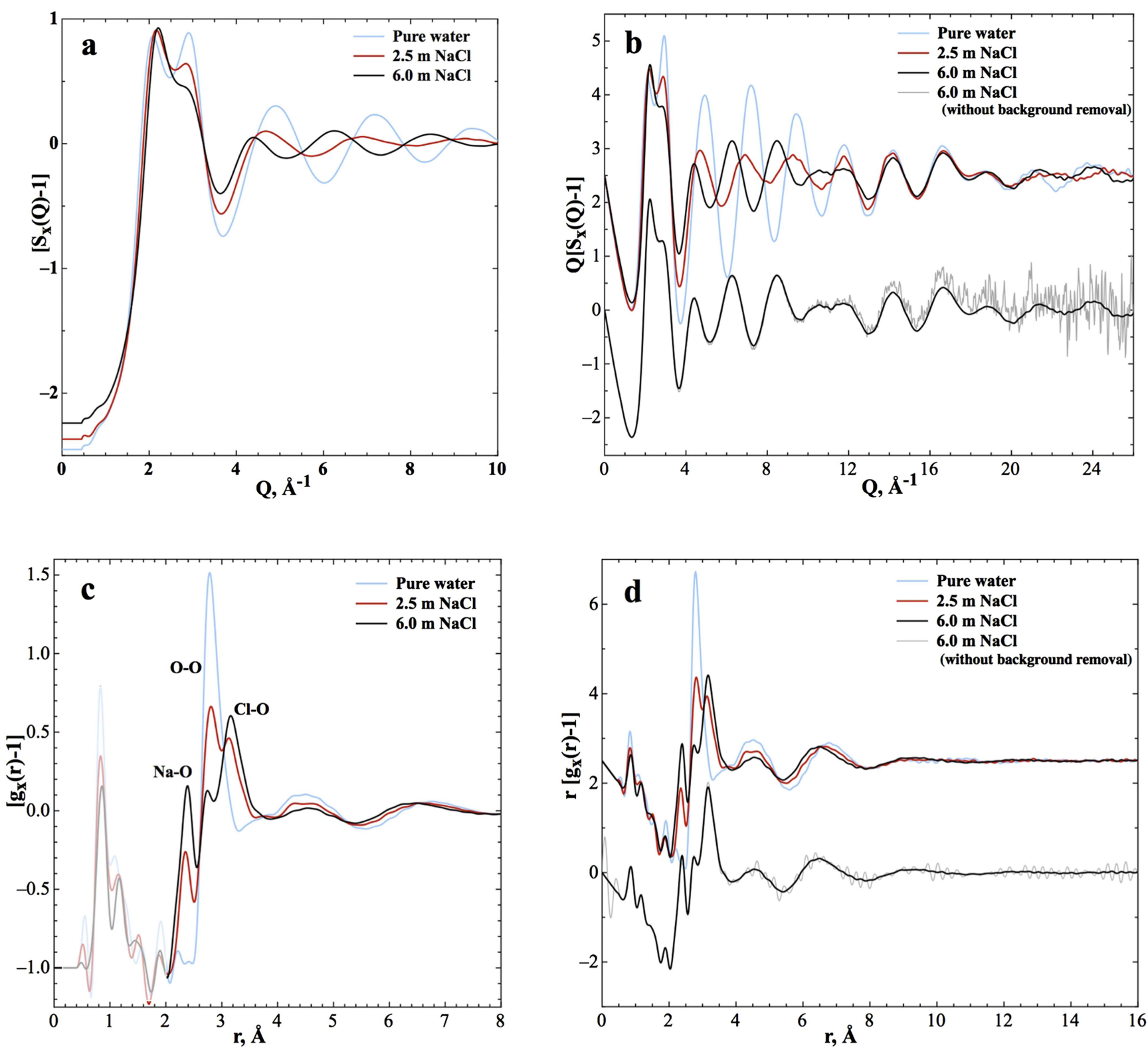

FIG. 7. The measured structure factors ((a) and (b)) and pair distribution functions ((c) and (d)) for two NaCl solutions (2.5 and 6 m) and for pure water. All measurements were made at room temperature. In b and d, the upper curves are the measured weighted x-ray patterns after optimization to remove the unphysical structure at $\mathrm{r}<1.3 \AA$ and removal of the high frequency Q noise. The lower curves show the $6.0 \mathrm{~m} \mathrm{NaCl}$ measurement with and without any optimization or smoothing (black and grey curves, respectively). In c, the region at $\mathrm{r}<2 \AA$ arises due to intramolecular water scattering. The plots have been offset by a value of 2.5 for clarity. 
$\mathrm{S}_{\mathrm{X}}(\mathrm{Q})$ and $\mathrm{g}_{\mathrm{X}}(\mathrm{r})$ (see Ref. 15 for details). For the $6.0 \mathrm{~m} \mathrm{NaCl}$ solution, the $\mathrm{x}$-ray spectra in both $\mathrm{S}(\mathrm{Q})$ and $\mathrm{g}(\mathrm{r})$ become dominated by the contributions from $\mathrm{Na}-\mathrm{O}, \mathrm{Cl}-\mathrm{O}$ with relatively weak proportional contribution from $\mathrm{O}-\mathrm{O}$ structure. Hence the dominant features of pure water spectra in Figures 7(a) and 7(c) are largely replaced by structure arising from cation-water and anion-water interactions.

In Figure 7(c), the primary peaks at about 2.35 and $3.2 \AA$ are due to the first-shell water about $\mathrm{Na}^{+}$and $\mathrm{Cl}^{-}$, respectively. The distinct $\mathrm{Na}-\mathrm{O}$ peak is different than previous XRD studies of this system because the spatial resolution, Q $\mathrm{Max}$ $=24 \AA^{-1}$, is much higher than in earlier studies. Three earlier XRD studies ${ }^{5-7}$ do not show a well-defined $\mathrm{Na}-\mathrm{O}$ peak since the resolution is limited by $\mathrm{Q}_{\mathrm{Max}}=16 \AA^{-1}$. The corresponding $\mathrm{Na}-\mathrm{O}$ distances in these earlier studies are 2.43 , 2.44, and $2.45 \AA$ for an average distance of about $2.44 \AA$. In general, most earlier XRD studies show a significantly longer
$\mathrm{Na}-\mathrm{O}$ distance than in this report. Table II shows that the $\mathrm{Na}-\mathrm{O}$ distance derived from the $g_{x}(r)$ in Figure $7(\mathrm{c})$ is $2.38 \AA$. This is in excellent agreement with the EXAFS value of $2.37 \AA$. The measured $\mathrm{Na}-\mathrm{O}$ coordination number from this XRD study is $5.5 \pm 0.3$.

Neutron diffraction with empirical potential structure refinement methods ${ }^{8}$ (EPSR) gives $\mathrm{Na}-\mathrm{O}$ distance of $2.34 \AA$ $\left(\mathrm{Q}_{\max }=16 \AA^{-1}\right.$ and $\left.\mathrm{CN}_{\mathrm{H}_{2} \mathrm{O}} 4.5(1.4)\right)$. A more direct method of ND scattering measurement ${ }^{9}$ involves removal of the waterwater by subtracting a null mixture ( $\mathrm{H}$ and $\mathrm{D})$ of pure water. This method gives an $\mathrm{Na}-\mathrm{O}$ distance of $2.34\left(\mathrm{CN}_{\mathrm{H}_{2} \mathrm{O}}\right.$ of 5.0 $(0.5))$.

\section{Comparing MD structure with XRD experiment}

The pure water $\mathrm{S}(\mathrm{Q})$ in Figure 8(a) is compared to predicted structure from revPBE-D3. In a prior publication, ${ }^{16}$ we have shown that this DFT variant quantitatively reproduces
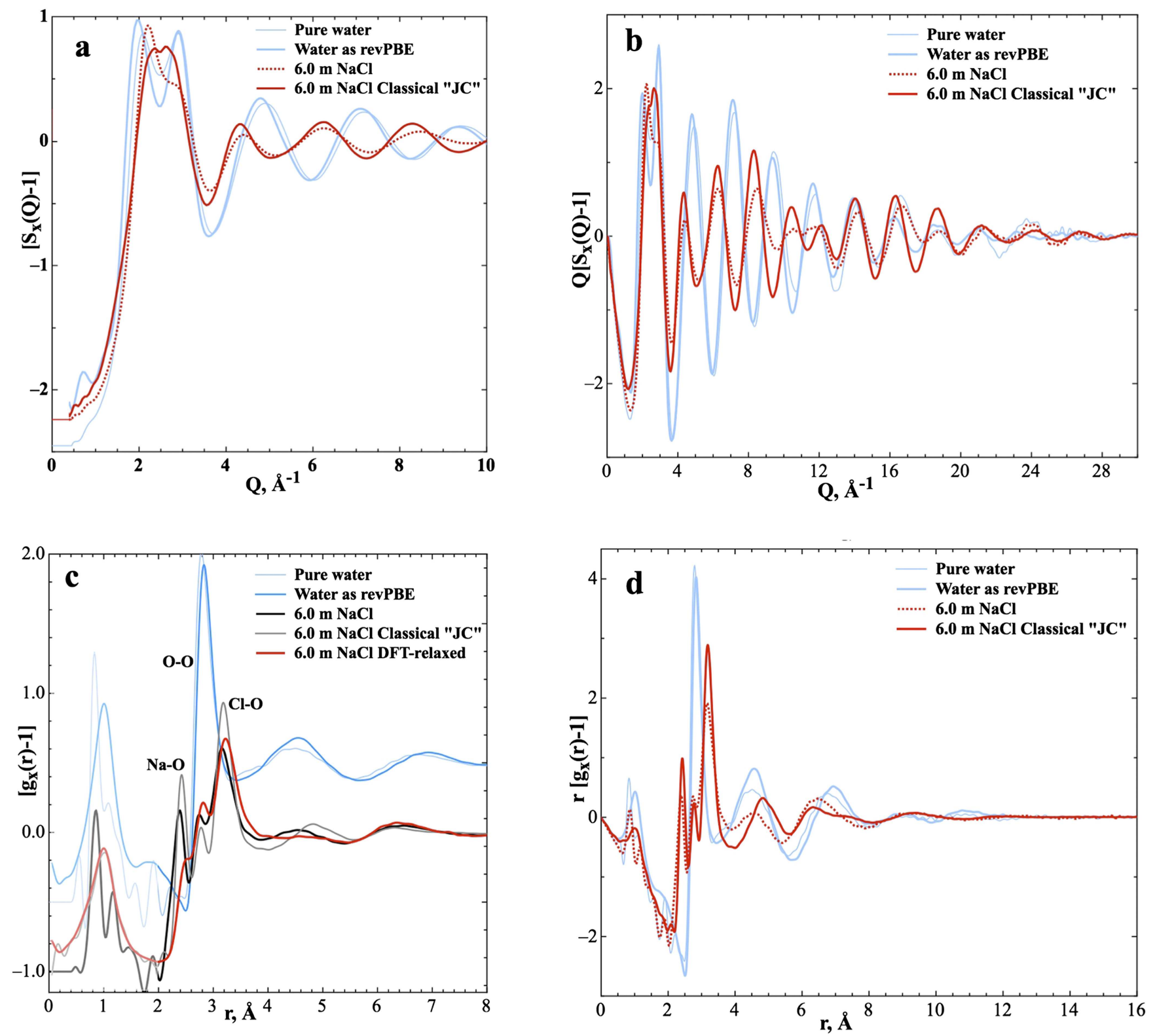

FIG. 8. The measured and predicted structure factors ((a) and (b)) and pair distribution functions ((c) and (d)) for $6 \mathrm{~m}$ NaCl and for pure water. In (c), the two pure water pair distribution functions have been offset by 0.5 for clarity. 

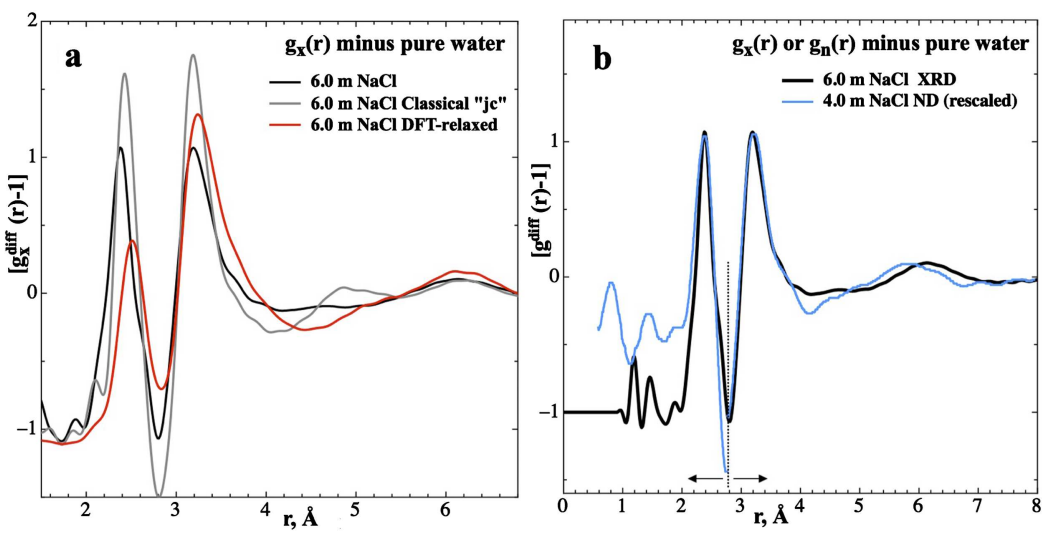

FIG. 9. Difference spectra of 6.0 or $4.0 \mathrm{~m} \mathrm{NaCl}$ minus the water-water structure approximated as the fractional contribution of pure water (for experimental XRD or ND and theoretical MD). (b) Comparison of the XRD spectrum with a neutron diffraction (ND) spectrum from Ref. 9. In both cases, the spectrum of pure water (XRD or ND) was used to remove the fractional contribution of water to the total $\mathrm{g}(\mathrm{r})$ in each sample. The ND spectrum has been rescaled in the follow way. The first $(\mathrm{Na}-\mathrm{O})$ and second $(\mathrm{Cl}-\mathrm{O})$ peaks have been partitioned (before and after dashed lines) and then the ND spectra in these two regions were rescaled to correct for different scattering factors (for Na, $31.0 \mathrm{fm}$ (XRD) or $3.63 \mathrm{fm}$ (ND) and for Cl, $47.9 \mathrm{fm}$ (XRD) or 9.58 fm (ND)). Finally a single scaling factor is applied to this modified $g_{n}(r)$ in order to match the overall amplitude of $g_{x}(r)$.

the structure better than the best available empirical potentials. This is true not only for pure water under ambient conditions but also at high pressure of $360 \mathrm{MPa}, 27^{\circ} \mathrm{C}$ and at high temperatures of $70^{\circ} \mathrm{C}$. The $\mathrm{S}(\mathrm{Q})$ in Figure 8 (a) for $6.0 \mathrm{~m} \mathrm{NaCl}$ is predicted using the classical "JC" potential. The major features of $S(Q)$ are replicated over the full Q-range, in which the frequency information is matched but the amplitudes are generally too high. It is notable that features in $\mathrm{S}(\mathrm{Q})$ up to $\mathrm{Q}_{\max }=28$ $\AA^{-1}$ are reproduced with this empirical potential model. In the $\mathrm{g}_{\mathrm{x}}(\mathrm{r})$ of Figure $8(\mathrm{c})$, the positions of the $\mathrm{Na}-\mathrm{O}$ and $\mathrm{Cl}-\mathrm{O}$ at 2.35 and $3.2 \AA$ are faithfully reproduced, although again, the peak heights are too high indicative of an over-structured system.

Also shown in Figure 8(c) is a treatment using DFT. Using the current DFT-MD methods, the ion diffusion times are too long in order to reach minimization of the longer-range $\mathrm{Na}^{+} / \mathrm{Cl}^{-}$structure in these highly concentrated solutions. Thus a simple approximation is used wherein the starting trajectory for this DFT simulation is chosen as the end point of the fully optimized JC-SPC/E trajectory. Then the last 25 ps of a $50 \mathrm{ps}$ DFT simulation were used to generate the "DFT-relaxed" $\mathrm{g}(\mathrm{r})$ in Figure $8(\mathrm{c})$. While the long range $\mathrm{Na}$ and $\mathrm{Cl}$ structure $(>5 \AA)$ is little changed from the "JC" configuration, the local structure about both the cation and anion has nearly completely relaxed to structures that are very similar to those in dilute systems. (The S(Q) for "DFT-relaxed" is given in the supplementary material.) DFT relaxed structure significantly improves the agreement with the $\mathrm{Cl}-\mathrm{O}$ peak around $3.2 \AA$ and the small water-water peak at $2.8 \AA$ but is substantially worse in predicting the $\mathrm{Na}-\mathrm{O}$ structure around $2.35 \AA$ than the JC empirical model. This is consistent with the over-prediction of the $\mathrm{Na}-\mathrm{O}$ distances with DFT that has been previously described in Secs. III A.

For the experimental XRD (and ND), an approximation can be used to remove the contribution of the water-water structure from the total $g(r)$ thus better highlighting the ion-water peaks in the $\mathrm{g}(\mathrm{r})$. In this process, the water-water correlations are removed by subtracting the fractional contribution of pure water using the $\mathrm{F}(\mathrm{Q})$ functions ${ }^{9,15,16}$ prior to generation of the $\mathrm{g}(\mathrm{r})$. This approach is similar to a method that has been previously described ${ }^{67,68}$ This is an approximation since it assumes that the water-water structure is not perturbed by the presence of the cations and anions. The merits of this approach can be tested with an MD trajectory where clean removal of the water-water contribution (with the salt) can be achieved.

Figure 9 illustrates this process for both the experimental and the simulated $\mathrm{g}(\mathrm{r}) \mathrm{s}$. In Figure 9(a), the experimental XRD spectra are now resolved with two distinct peaks, a rather narrow Gaussian like peak for the $\mathrm{Na}-\mathrm{O}$ structure at $2.38 \AA$ and a Cl-O peak at $3.2 \AA$ having a tailing structure on the longdistance side of the distribution from approximately 3.2-4.0. . The exact same treatment was applied to the empirical JC and the DFT-relaxed structure in that, for each case, simulations of pure water were used in the background subtraction. This process highlights the strengths and weaknesses of the classical and DFT-based potentials.

While the use of pure water represents only an approximation as mentioned above, an exact difference spectrum can be obtained from the MD simulation by using the $g_{O O}(r)$ generated from trajectory of the $6.0 \mathrm{~m} \mathrm{NaCl}$ solution (results shown in the supplementary material). This comparison relates the favorable outcome, in that the experimental $\mathrm{Na}-\mathrm{O}$ peak at $2.38 \AA$ is sufficiently far away from the water-water structure at $2.8 \AA$, that there is little distortion of the $\mathrm{Na}-\mathrm{O}$ peak upon subtraction of the pure water-water structure in the experimental system.

In Figure 9(b), a further comparison is made to earlier set of neutron diffraction data ${ }^{9}$ that employs effectively the same water-water difference method that was used in the XRD study. In Figure 9(b), the $\mathrm{Na}-\mathrm{O}$ and the $\mathrm{Cl}-\mathrm{O}$ peaks are rescaled to normalize the ND scattering factor contributions to those matching the XRD values. The resultant XRD and ND g(r)'s are remarkably similar in both the position and shape of the $\mathrm{Na}-\mathrm{O}$ and $\mathrm{Cl}-\mathrm{O}$ peaks.

\section{CONCLUSIONS}

We have investigated the hydration structure of $\mathrm{Na}^{+}$ in water by using both experimental and computational methods. The XRD results, using a high spatial resolution corresponding to $\mathrm{Q}_{\max }=24 \AA^{-1}$, provided a Na-O distance of 
$2.384 \pm 0.003 \AA$ A In addition to XRD, Na K EXAFS measurements using a spatial resolution corresponding to $\mathrm{Q}_{\max }=16$ $\AA^{-1}$ provided a Na-O distance of $2.37 \pm 0.024 \AA$. Hence the XRD and EXAFS measurements carried out in this study provide consistent results. The values of $\mathrm{Na}-\mathrm{O}$ distance obtained here are much shorter than those usually reported in the experimental XRD literature. Interestingly a recent neutron diffraction measurement ${ }^{9}$ provided a $\mathrm{Na}-\mathrm{O}$ distance which is close to the results presented here. The $\mathrm{Na}^{+}$-water coordination number was measured at $5.5 \pm 0.3$ from the XRD measurement.

We carried out first-principles molecular dynamics simulations using the revPBE functional along with Grimme D3 dispersion correction. Even though this DFT protocol was previously found to be very good at reproducing the experimental structure of water, the $\mathrm{Na}-\mathrm{O}$ distance was found to be overestimated by more than $0.1 \AA$. The overestimation of $\mathrm{Na}-\mathrm{O}$ distance within the revPBE-D3 level of theory is consistent with the other DFT functionals that have been reported previously, e.g., BLYP-D2, PBE-D2, and HCTH-D2. This indicates that the GGA functional, even with Grimme dispersion, inadequately describes this monovalent cation hydration in water.

We have also found that the use of dispersion correction has a significant impact on both the $\mathrm{Na}-\mathrm{O}$ distance and the coordination number. In the absence of dispersion correction, the $\mathrm{Na}-\mathrm{O}$ distance is shorter by $0.08-0.10 \AA$, thus closer to the experimental value. However, without including dispersion, the water-water interactions are certainly poorly described. The coordination number also moved from 5.7 to 6.0-6.1 when dispersion is added. The free energy calculations show, that for BLYP functional, the 6-coordinated local structure is the most stable one when dispersion is included. However, in the absence of dispersion, the most stable energy well corresponds to a coordination number of 5.2. Both of these two local structures are within $2 \mathrm{kcal} / \mathrm{mol}$ of each other and thus contribute some populations of each local structure in an equilibrium. We have simulated the K-edge XANES spectra of aqueous $\mathrm{Na}^{+}$for two separate ensembles of configurations, one corresponding to a coordination number of 5 and the other to 6 . Both ensembles reproduced the features in the experimental spectra. There were no significant differences between the spectra for these two ensembles indicating that the K-edge XANES cannot be used to quantitatively differentiate the coordination number in the first solvation shell of $\mathrm{Na}^{+}$.

We also carried out classical molecular dynamics simulations using JC force field and SPC/E water which provided a $\mathrm{Na}-\mathrm{O}$ distance of $2.39 \AA$ $\mathrm{\text {that }}$ is an excellent agreement with the experiments. This is consistent with the previously reported values in literature such as the Dang polarizable model that provided a Na-O distance of $2.34 \AA$ A. Since classical models are directly parameterized to the experimental properties, they are thus usually good at describing the single ion hydration structure satisfactorily.

\section{SUPPLEMENTARY MATERIAL}

See the supplementary material for the structure factors for all simulations, the simulated water pair distribution functions, and the difference spectra for $6 \mathrm{~m} \mathrm{NaCl}$.

\section{ACKNOWLEDGMENTS}

This work was supported by the U.S. Department of Energy (DOE) office of Basic Energy Sciences Grant No. BES DE-FG02-09ER46650 which supported data analysis and manuscript preparation (L.B.S.). DOE Contract No. DEAC02-06CH11357 supports operation of the Advanced Photon Source at Argonne National Laboratory. Work by J.L.F., M.G., N.G., G.K.S., and C.J.M. was supported by the U.S. Department of Energy, Office of Science, Office of Basic Energy Sciences, Division of Chemical Sciences, Geosciences \& Biosciences. Pacific Northwest National Laboratory (PNNL) is a multiprogram national laboratory operated for DOE by Battelle. The Al XAFS measurements were performed at the PHOENIX beamline of the Swiss Light Source, Paul Scherrer Institute, Villigen, Switzerland. This research also used resources of the National Energy Research Scientific Computing Center, a DOE Office of Science User Facility supported by the Office of Science of the U.S. Department of Energy under Contract No. DE-AC02-05CH11231. This research also benefited from computer resources provided by PNNL Institutional Computing (PIC) and EMSL, a DOE Office of Science User Facility sponsored by the Office of Biological and Environmental Research and located at PNNL. PNNL is operated by Battelle Memorial Institute for the United States Department of Energy under DOE Contract No. DE-AC05-76RL1830.

${ }^{1}$ M. Soniat, D. M. Rogers, and S. B. Rempe, J. Chem. Theory Comput. 11(7), 2958-2967 (2015).

${ }^{2}$ A. P. Gaiduk, C. Zhang, F. Gygi, and G. Galli, Chem. Phys. Lett. 604, 89-96 (2014).

${ }^{3}$ A. Bankura, V. Carnevale, and M. L. Klein, J. Chem. Phys. 138(1), 014501 (2013).

${ }^{4}$ A. Bankura, V. Carnevale, and M. L. Klein, Mol. Phys. 112(9-10), 1448-1456 (2014).

5 J. Mahler and I. Persson, Inorg. Chem. 51(1), 425-438 (2012).

${ }^{6}$ R. Caminiti, G. Licheri, G. Paschina, G. Piccaluga, and G. Pinna, J. Chem. Phys. 72(8), 4522-4528 (1980).

${ }^{7}$ T. Megyes, S. Balint, T. Grosz, T. Radnai, I. Bako, and P. Sipos, J. Chem. Phys. 128(4), 044501 (2008).

${ }^{8}$ R. Mancinelli, A. Botti, F. Bruni, M. A. Ricci, and A. K. Soper, J. Phys. Chem. B 111(48), 13570-13577 (2007).

${ }^{9}$ P. E. Mason, S. Ansell, and G. W. Neilson, J. Phys.: Condens. Matter 18(37), 8437-8447 (2006).

${ }^{10}$ J. L. Fulton, E. J. Bylaska, S. Bogatko, M. Balasubramanian, E. Cauet, G. K. Schenter, and J. H. Weare, Phys. Chem. Lett. 3(18), 2588-2593 (2012).

${ }^{11}$ J. L. Fulton, G. K. Schenter, M. D. Baer, C. J. Mundy, L. X. Dang, and M. Balasubramanian, J. Phys. Chem. B 114(40), 12926-12937 (2010).

${ }^{12}$ M. D. Baer, J. L. Fulton, M. Balasubramanian, G. K. Schenter, and C. J. Mundy, J. Phys. Chem. B 118(26), 7211-7220 (2014).

${ }^{13}$ J. L. Fulton, S. M. Kathmann, G. K. Schenter, and M. Balasubramanian, J. Phys. Chem. A 113(50), 13976-13984 (2009).

${ }^{14}$ E. R. Johnson, J. Chem. Phys. 135(23) (2011).

${ }^{15}$ L. B. Skinner, C. Huang, D. Schlesinger, L. G. M. Pettersson, A. Nilsson, and C. J. Benmore, J. Chem. Phys. 138(7), 074506 (2013).

${ }^{16}$ L. B. Skinner, M. Galib, J. L. Fulton, C. J. Mundy, J. B. Parise, V.-T. Pham, G. K. Schenter, and C. J. Benmore, J. Chem. Phys. 144, 134504 (2016).

${ }^{17}$ V.-A. Glezakou, Y. S. Chen, J. L. Fulton, G. K. Schenter, and L. X. Dang, Theor. Chem. Acc. 115, 86-99 (2006).

${ }^{18}$ L. X. Dang, G. K. Schenter, V. A. Glezakou, and J. L. Fulton, J. Phys. Chem. B 110(47), 23644-23654 (2006).

${ }^{19}$ J. L. Fulton and M. Balasubramanian, J. Am. Chem. Soc. 132(36), 12597-12604 (2010).

${ }^{20}$ J. L. Fulton, N. Govind, T. Huthwelker, E. J. Bylaska, A. Vjunov, S. Pin, and T. D. Smurthwaite, J. Phys. Chem. B 119(26), 8380-8388 (2015).

${ }^{21}$ A. A. Hassanali, J. Cuny, V. Verdolino, and M. Parrinello, Philos. Trans. R. Soc., A 372(2011), 20120482 (2014). 
${ }^{22}$ A. Bankura, A. Karmakar, V. Carnevale, A. Chandra, and M. L. Klein, Phys. Chem. C 118(50), 29401-29411 (2014).

${ }^{23}$ M. Del Ben, M. Schonherr, J. Hutter, and J. VandeVondele, Phys. Chem. Lett. 4(21), 3753-3759 (2013).

${ }^{24}$ M. F. Harrach and B. Drossel, J. Chem. Phys. 140(17) (2014).

${ }^{25}$ Y. Ding, A. A. Hassanali, and M. Parrinello, Proc. Natl. Acad. Sci. U. S. A. 111(9), 3310-3315 (2014).

${ }^{26}$ Y. Yao, Y. Kanai, and M. L. Berkowitz, Phys. Chem. Lett. 5(15), 2711-2716 (2014).

${ }^{27}$ M. D. Baer and C. J. Mundy, J. Phys. Chem. B 120(8), 1885-1893 (2016).

${ }^{28}$ J. Klimes and A. Michaelides, J. Chem. Phys. 137(12), 120901 (2012).

${ }^{29}$ S. Grimme, Wiley Interdiscip. Rev.: Comput. Mol. Sci. 1(2), 211-228 (2011).

${ }^{30}$ A. D. Becke, Phys. Rev. A 38(6), 3098-3100 (1988).

${ }^{31}$ C. T. Lee, W. T. Yang, and R. G. Parr, Phys. Rev. B 37(2), 785-789 (1988).

32 J. P. Perdew, K. Burke, and M. Ernzerhof, Phys. Rev. Lett. 77(18), 3865-3868 (1996).

${ }^{33}$ S. Grimme, J. Comput. Chem. 27(15), 1787-1799 (2006).

${ }^{34}$ C. Adamo and V. Barone, J. Chem. Phys. 110(13), 6158-6170 (1999).

${ }^{35}$ C. Zhang, D. Donadio, F. Gygi, and G. Galli, J. Chem. Theory Comput. 7(5), 1443-1449 (2011).

${ }^{36}$ Y. K. Zhang and W. T. Yang, Phys. Rev. Lett. 80(4), 890-890 (1998).

${ }^{37}$ S. Grimme, J. Antony, S. Ehrlich, and H. Krieg, J. Chem. Phys. 132(15), 154104 (2010).

${ }^{38}$ I. S. Joung and T. E. Cheatham, J. Phys. Chem. B 112(30), 9020-9041 (2008).

${ }^{39}$ H. J. C. Berendsen, J. R. Grigera, and T. P. Straatsma, J. Phys. Chem. 91(24), 6269-6271 (1987).

${ }^{40}$ C. Piamonteze, U. Flechsig, S. Rusponi, J. Dreiser, J. Heidler, M. Schmidt, R. Wetter, M. Calvi, T. Schmidt, H. Pruchova, J. Krempasky, C. Quitmann, H. Brune, and F. Nolting, J. Synchrotron Radiat. 19, 661-674 (2012).

${ }^{41}$ M. Newville, J. Synchrotron Radiat. 8, 322-324 (2001).

${ }^{42}$ B. Ravel and M. Newville, J. Synchrotron Radiat. 12, 537-541 (2005).

${ }^{43}$ E. A. Stern, M. Newville, B. Ravel, Y. Yacoby, and D. Haskel, Phys. B 208-209, 117-120 (1995).

${ }^{44}$ S. I. Zabinsky, J. J. Rehr, A. Ankudinov, R. C. Albers, and M. J. Eller, Phys. Rev. B 52, 2995-3009 (1995).

${ }^{45}$ J. J. Rehr and R. C. Albers, Rev. Mod. Phys. 72(3), 621-654 (2000).
${ }^{46}$ A. P. Hammersley, ESRF Internal Report ESRF97HA02T, 1997.

${ }^{47}$ J. VandeVondele, M. Krack, F. Mohamed, M. Parrinello, T. Chassaing, and J. Hutter, Comput. Phys. Commun. 167(2), 103-128 (2005).

${ }^{48}$ G. J. Martyna, M. L. Klein, and M. Tuckerman, J. Chem. Phys. 97(4), 2635-2643 (1992).

${ }^{49}$ S. Goedecker, M. Teter, and J. Hutter, Phys. Rev. B 54(3), 1703-1710 (1996).

${ }^{50}$ F. Moucka, I. Nezbeda, and W. R. Smith, J. Chem. Phys. 138(15), 154102 (2013).

${ }^{51}$ U. Essmann, L. Perera, M. L. Berkowitz, T. Darden, H. Lee, and L. G. Pedersen, J. Chem. Phys. 103(19), 8577-8593 (1995).

${ }^{52}$ D. Waasmaier and A. Kirfel, Acta Crystallogr., Sect. A 51, 416-431 (1995).

${ }^{53}$ K. Lopata, B. E. Van Kuiken, M. Khalil, and N. Govind, J. Chem. Theory Comput. 8(9), 3284-3292 (2012).

${ }^{54}$ M. Valiev, E. J. Bylaska, N. Govind, K. Kowalski, T. P. Straatsma, H. J. J. Van Dam, D. Wang, J. Nieplocha, E. Apra, T. L. Windus, and W. de Jong, Comput. Phys. Commun. 181(9), 1477-1489 (2010).

${ }^{55}$ T. Noro, M. Sekiya, and T. Koga, Theor. Chem. Acc. 131(2), 1-8 (2012).

${ }^{56}$ R. Krishnan, J. S. Binkley, R. Seeger, and J. A. Pople, J. Chem. Phys. 72(1), 650-654 (1980).

${ }^{57}$ A. D. Becke, J. Chem. Phys. 98(2), 1372-1377 (1993).

${ }^{58}$ J. Schmidt, J. VandeVondele, I. F. W. Kuo, D. Sebastiani, J. I. Siepmann, J. Hutter, and C. J. Mundy, J. Phys. Chem. B 113(35), 11959-11964 (2009).

${ }^{59}$ M. Krack and M. Parrinello, PCCP 2(10), 2105-2112 (2000).

${ }^{60}$ D. E. Smith and L. X. Dang, J. Chem. Phys. 100(5), 3757-3766 (1994).

${ }^{61}$ J. A. White, E. Schwegler, G. Galli, and F. Gygi, J. Chem. Phys. 113(11), 4668-4673 (2000).

${ }^{62}$ S. B. Rempe and L. R. Pratt, Fluid Phase Equilib. 183, 121-132 (2001).

${ }^{63}$ S. Varma and S. B. Rempe, Biophys. Chem. 124(3), 192-199 (2006).

${ }^{64}$ D. M. Rogers, D. Jiao, L. R. Pratt and S. B. Rempe, in Annual Reports in Computational Chemistry, edited by R. A. Wheeler (Elsevier, Amsterdam, 2012), Vol. 8, pp. 71-128.

${ }^{65}$ S. B. Rempe, T. R. Mattsson, and K. Leung, PCCP 10(32), 4685-4687 (2008).

${ }^{66}$ E. F. Aziz, A. Zimina, M. Freiwald, S. Eisebitt, and W. Eberhardt, J. Chem. Phys. 124(11), 114502 (2006).

${ }^{67}$ J. Neuefeind, S. Skanthakumar, and L. Soderholm, Inorg. Chem. 43(7), 2422-2426 (2004).

${ }^{68}$ J. Neuefeind, L. Soderholm, and S. Skanthakumar, J. Phys. Chem. A 108(14), 2733-2739 (2004). 